\title{
REFERENCES
}

1. N. Muschelišvili, Praktische Lösung der fundamentalen Randwertaufgaben der Elastizitätstheorie in der Ebene für einige Berandungsformen, ZaMM 13, 264-282 (1933).

2. N. Muschelišvili, Recherches sur les problèmes aux limites relatifs à l'équation biharmonique et aux equations de l'élasticité à deux dimensions, Math. Annalen 107, 282-312 (1933).

3. S. Timoshenko, Theory of elasticity, McGraw-Hill, New York, 1934, pp. 25-26.

4. H. Love, Mathematical theory of elasticity, 4th ed., 1927, p. 219.

5. G. Horvay and H. Poritsky, Gravitational stresses in a disk supported at the ends of the horizontal diameter, General Electric, Knolls Atomic Power Lab., Schenectady, N. Y., 1951.

\section{REPRESENTATION OF NONLINEAR FIELD FUNCTIONS BY THIELE SEMI-INVARIANTS ${ }^{1}$}

\author{
BY WILLIAM M. MACDONALD, III, ${ }^{2}$ JOHN M. RICHARDSON, ${ }^{3}$ \\ AND LEON P. ROSENBERRY ${ }^{4}$
}

1. Certain nonlinear field functions, possessing the property of space localization of the gradients of the dependent variables, occur in the problem of flame propagation in continuous media. The difficulties encountered in solving the equations of propagation may be considerably diminished by introducing new dependent variables (intrinsically connected with this localization) within a certain region, outside of which the equations may be linearized and treated in a point-wise sense by well-known methods. Specifically, we choose the $x$-axis as nearly perpendicular to the flame front and define the region as $x_{1}(y, z) \leqq x \leqq x_{2}(y, z)$. Taking a state variable, temperature $T$, for example, we choose the new dependent variables as the $\kappa_{\nu}$ given by

$$
\sum_{\nu=0}^{\infty}(i t)^{\nu} \frac{\kappa_{\nu}}{\nu !}=\log [(\phi t)]
$$

where

$$
\phi(t)=\int_{x_{1}}^{x_{2}} d x e^{i t x} \frac{\partial T}{\partial x}
$$

These definitions are closely related to the formalism of mathematical statistics. In particular, if one considers $\partial T / \partial x$ to correspond to an unnormalized distribution function with range $\left(x_{1} \leqq x \leqq x_{2}\right)$, the $\kappa_{\nu}$ correspond to the Thiele semi-invariants [1] of $\partial T / \partial x$ and completely describe a given function in the range $\left(x_{1} \leqq x \leqq x_{2}\right)$.

Received Oct. 8, 1951.

${ }^{1}$ This research is part of the work being done by the Bureau of Mines on Contract NA onr 25-47, NR 090 117, (33-038) 51-4151, supported by the Office of Naval Research and the Air Materiel Command.

${ }^{2}$ Formerly Physicist, Mathematical and Theoretical Physics Section, Explosives and Physical Sciences Division, Bureau of Mines, Pittsburgh, Pa. Present åddress: Department of Physics, Princeton University.

${ }^{3}$ Head, Kinetics Section, Explosives and Physical Sciences Division, Bureau of Mines, Pittsburgh, Pa.

${ }^{4}$ Physicist, Mathematical and Theoretical Physics Section, Explosives and Physical Sciences Division, Pittsburgh, Pa. 
The semi-invariants were chosen (in preference to the moments

$$
\int_{x_{1}}^{x_{2}} d x x^{\nu} \frac{\partial T}{\partial x}
$$

for example) because evaluation of the integrals

$$
\int_{x_{1}}^{x_{2}} d x x^{n} T^{s} \frac{\partial T}{\partial x}
$$

led to application of the Fourier inversion formula to the characteristic function (1.2), where the exponential form considerably lessened the difficulties involved. The investigation was extended to include integrals of the form

$$
\int_{x_{1}}^{x_{2}} d x x^{n} T_{1} T_{2} \cdots T_{m} \frac{\partial T_{m+1}}{\partial x}
$$

2. The characteristic function $\phi(t)$ of $\partial T / \partial x$, in the region $x_{1}(y, z) \leqq x \leqq x_{2}(y, z)$ is given by Eq. (1.2). Using the Fourier inversion formula one finds

$$
\begin{aligned}
\frac{1}{2 \pi} \int_{-\infty}^{\infty} d t e^{-i t x} \phi(t) & =\frac{\partial T}{\partial x}, \quad x_{1} \leqq x \leqq x_{2} \\
& =0, \quad \text { otherwise, }
\end{aligned}
$$

from which the following expression for $T$ can be obtained if $\partial T / \partial x$ satisfies certain mild restrictions [2]:

$$
T(x)-T\left(x_{1}\right)=\left(\frac{i}{2 \pi}\right) \int_{-\infty}^{\infty} d t\left(e^{-i t x}-e^{-i t x_{1}}\right) t^{-1} \phi(t) d t
$$

Writing

$$
\phi(t) e^{-i t x}=\int_{x_{1}}^{x_{2}} d \xi\left(\frac{\partial T}{\partial \xi}\right) \exp \left[i t\left(\xi-x_{1}\right)\right],
$$

it can be seen that, for $\partial T / \partial x$ bounded, $\phi(t) e^{-i t x}$ has no poles or other singularities other than an essential singularity at infinity and that here

$$
\phi(t) e^{-i t x} \rightarrow 0 \text { as } t \rightarrow \infty \text {, with }-\pi / 2<\arg t<\pi / 2 .
$$

We use this fact to reduce Eq. (2.2) to a more suitable form by writing

$$
\int_{-\infty}^{\infty} d t\left(e^{-i t x}-e^{-i t x_{1}}\right) t^{-1} \phi(t)=P \int_{-\infty}^{\infty} d t \phi(t) e^{-i t x} t^{-1}-P \int_{-\infty}^{\infty} d t \phi(t) e^{-i t x_{1}} t^{-1}
$$

where $P \int_{-\infty}^{\infty} \cdots$ denotes the Cauchy proper value

$$
\operatorname{Lim}_{\epsilon \rightarrow 0}\left[\int_{-\infty}^{-\epsilon}+\int_{\epsilon}^{\infty}\right]_{\lambda} .
$$

Contour integration and application of the theory of residues then yields for the last integral on the right of (2.4) the expression

$$
P \int_{-\infty}^{\infty} d t e^{-i t x_{1}} t^{-1} \phi(t)=i \pi \phi(0)
$$


If $\Im(x)$ is defined as

$$
\Im(x)=T(x)-\frac{1}{2}\left[T\left(x_{1}\right)+T^{\prime}\left(x_{2}\right)\right],
$$

then from Eqs. (1.2), (2.2) and (2.5), one obtains

$$
\Im(x)=\left(\frac{i}{2 \pi}\right) \int_{-\infty}^{\infty} d t e^{-i t x} t^{-1} \phi(t)
$$

and

$$
\frac{\partial \Im(x)}{\partial x}=\left(\frac{1}{2 \pi}\right) \int_{-\infty}^{\infty} d t e^{-i t x} \phi(t) .
$$

In Eqs. (2.7) and (2.8) and henceforth we conventionally denote $P \int_{-\infty}^{\infty}$ by $\int_{-\infty}^{\infty}$.

3 . We now apply (2.7) and (2.8) to

$$
\phi_{s}(t)=\int_{-\infty}^{\infty} d x e^{i t x} \mathcal{Y}^{s} \frac{\partial \mathfrak{Y}}{\partial x},
$$

obtaining

$$
\phi_{s}(t)=i^{s}(2 \pi)^{-s-1} \int_{-\infty}^{\infty} d x e^{i t x}\left[\left\{\int_{-\infty}^{\infty} d t e^{-i t x} t^{-1} \phi(t)\right\}^{s} \int_{-\infty}^{\infty} d t e^{-i t x} \phi(t)\right] .
$$

Using independent variables $t_{0}, t_{1}, \cdots, t_{s}$, one can write

$\phi_{s}(t)=i^{s}(2 \pi)^{-s-1} \int_{-\infty}^{\infty} d x \int_{-\infty}^{\infty} d t_{s} \cdots \int_{-\infty}^{\infty} d t_{0} \prod_{r=0}^{s}\left[t_{r}^{-1} \phi\left(t_{r}\right)\right] \phi\left(t_{0}\right) \exp i x \sigma_{s}$,

$$
\text { where } \sigma_{s}(t)=t-\sum_{1}^{s} t_{a}
$$

Since $(2 \pi)^{-1} \int_{-\infty}^{\infty} d x e^{i x u}=\delta(u)$, one can write

$$
\phi_{s}(t)=i^{s}(2 \pi)^{-s-1} \int_{-\infty}^{\infty} d t_{s} \cdots \int_{-\infty}^{\infty} d t_{1} \prod_{r=1}^{s} t_{r}^{-1} \phi\left(t_{r}\right) \phi\left(\sigma_{s}\right) .
$$

To evaluate

$$
\mu_{\nu}=\int_{-\infty}^{\infty} d x x^{\nu} \Im^{s} \frac{\partial \Im}{\partial x}
$$

one notes that

$$
\phi^{(\nu)}(0)=\left(d^{\nu} \phi / d t^{\nu}\right)_{t=0}=\alpha_{\nu}=i^{\nu} \mu_{\nu}
$$

and hence that

$$
\phi_{s}(t)=\sum_{\nu=0}^{\infty} \alpha_{\nu} t^{\nu} / \nu !
$$

The $\alpha_{\nu}$ cannot be obtained in analytical form but one can easily expand $\alpha_{\nu}$ in a Taylor's series

$$
\begin{aligned}
& \alpha_{\nu}\left(\kappa_{0}, \kappa_{1}, \cdots, \kappa_{i}, \cdots\right) \\
& =\left(\alpha_{\nu}\right)_{P}+\sum_{i=3}^{\infty}\left(\frac{\partial \alpha_{\nu}}{\partial \kappa_{i}}\right)_{P} \kappa_{i}+\frac{1}{2} \sum_{i=3}^{\infty} \sum_{j=3}^{\infty}\left(\frac{\partial^{2} \alpha_{\nu}}{\partial \kappa_{i} \partial \kappa_{j}}\right)_{P} \kappa_{i} \kappa_{i}+\cdots
\end{aligned}
$$


about the point $P\left(\kappa_{0}, \kappa_{1}, \kappa_{2}, 0,0,0 \cdots\right)$ in $\kappa$-space ( $\kappa_{i}$ defined by Eq. (1.1)). The coefficients in this expansion are found to be

$$
\begin{aligned}
\left(\alpha_{v}\right)_{P} & =A_{0,0}^{s}(-1)^{\nu}\left(\kappa_{2} / 2\right)^{\nu / 2} J_{s} H_{\nu}\left(\tau_{s}\right), \\
\left(\frac{\partial \alpha_{v}}{\partial \kappa_{\sigma}}\right)_{P} & =A_{\sigma, 0}^{s} \Im_{s}\left[S_{\nu, \sigma}+\left(\sum_{p=1}^{s} t_{p}^{\sigma}\right) S_{\nu, 0}\right],
\end{aligned}
$$

and

$$
\left(\frac{\partial^{2} \alpha_{\nu}}{\partial \kappa_{\sigma} \partial \kappa_{\mu}}\right)_{P}=A_{\sigma, \mu}^{s} J_{s}\left[S_{\nu, \sigma+\mu}+S_{\nu \sigma} \sum_{p=1}^{s} t_{p}^{\sigma}+S_{\nu \mu} \sum_{p=1}^{s} t_{p}^{\mu}+S_{\nu 0}\left(\sum_{p=1}^{s} t_{p}\right)^{2}\right]
$$

where the following notation has been introduced:

$$
\begin{gathered}
A_{\sigma, \mu}^{s}=(i / 2 \pi)^{s} i^{\sigma+\mu} / \sigma ! \mu ! \\
J_{s}=e^{-(s+1) \kappa_{0}} \int_{-\infty}^{\infty} d t_{s} \cdots \int_{-\infty}^{\infty} d t_{1} \prod_{r=1}^{s}\left[\phi\left(t_{r}\right) / t_{r}\right] \phi\left(-\sum_{a=1}^{s} t_{a}\right) \text { an operator, } \\
S_{\nu \sigma}=\left[\left\{\xi^{\sigma} \phi_{P}(\xi) / \phi_{P}(\xi)\right\}^{(\nu)}\right]_{t=0},
\end{gathered}
$$

and $H_{\nu}\left(\tau_{s}\right)$ is the $\nu$ th Hermite polynomial.

4. Treatment of the equations of hydrodynamics by the moment transformation will clearly involve integrals in which the integrand includes various combinations of the gradient of a dependent variable and some other variable. We must therefore generalize the foregoing treatment to the case where $\Im$ is not merely the temperature but a generalized vector in a space of $n$ dimensions; this fact can be represented symbolically by writing

$$
\Im=\left(\Im_{1}, \Im_{2}, \cdots, \Im_{n}\right),
$$

where the product of two vectors $\Im$ and $\eta$ gives a vector in the product space of $\Im$ and $\eta$, or in our notation,

$$
\Im \eta=\left(\Im_{i} \eta_{i}\right)\left(\begin{array}{l}
i=1,2, \cdots, n \\
j=1,2, \cdots, n
\end{array}\right) .
$$

Designating by $\psi(t)$ the characteristic function of the component $\Im_{i}$ of $\Im$, one finds, as in section 2 , that from

$$
\psi_{i}(t)=\exp \left\{\sum_{\nu=0}^{\infty} \kappa_{i \nu} \frac{(i t)^{\nu}}{\nu !}\right\}=\int_{-\infty}^{\infty} d x e^{i t x} \frac{\partial \Im_{i}}{\partial x},
$$

follows

$$
\frac{\partial \Im_{i}}{\partial x}=\left(\frac{1}{2 \pi}\right) \int_{-\infty}^{\infty} d t e^{-i t x} \psi_{i}(t)
$$

and

$$
\Im_{i}=\left(\frac{i}{2 \pi}\right) \int_{-\infty}^{\infty} d t t^{-1} e^{-i t x} \psi_{i}(t)
$$


Here, $\Im_{i}$ is again the deviation from the average over the infinite range of $x$ of the corresponding dependent variable. The canonical integral

$$
\int_{-\infty}^{\infty} d x e^{i t x} \Im^{m} \frac{\partial \Im}{\partial x}
$$

represents then the characteristic function $\phi(t)$ whose components are given by the equation

$$
\phi_{a_{1}, a_{2}, \cdots, a_{m+1}}(t)=\int_{-\infty}^{\infty} d x e^{i \ell x} \Im_{a_{1}} \Im_{a 2} \cdots \Im_{a_{m}} \frac{\partial \Im_{a_{m+1}}}{\partial x},
$$

which is easily transformed by (4.5) into the form

$$
\phi_{a_{1}, a_{2}, \cdots, a_{m+1}}(t)=\left(\frac{i}{2 \pi}\right)^{m} \int_{-\infty}^{\infty} d t_{a_{1}} \cdots \int_{-\infty}^{\infty} d t_{a_{m}}\left(\prod_{i=1}^{m} \Psi_{a \kappa}\left(t_{i}\right) / t_{i}\right) \psi_{a_{m+1}}\left(\xi_{m}\right)
$$

where $\xi_{m}=t-\sum_{q=1}^{m} t_{q}$. We write, as before,

$$
\phi_{a_{1}, \cdots_{1} a_{m+1}}(t)=\sum_{\nu=0}^{\infty} \alpha_{\nu}^{a_{2} a_{2} \cdots a_{m+1}}(i t)^{\nu} / \nu !
$$

where

$$
\alpha_{\nu}^{a_{1} a_{3} \cdots a_{m+1}}=\phi_{a_{1}, a_{2}, \cdots, a_{m+1}}^{(\nu)}(0),
$$

the coefficients of this series then being found by a Taylor's series expansion of the form

$$
\alpha_{\nu}^{a_{2} a_{2} \cdots a_{m+1}}=\left(\alpha_{\nu}^{a_{1} a_{2} \cdots a_{m+1}}\right)_{P}+\exp \left[\left(\sum_{n=0}^{\infty} \sum_{i=1}^{m} \kappa_{a_{i n},} \frac{\partial}{\partial \kappa_{a i n}}\right)\left(\alpha_{\nu}^{a_{1} a_{2} \cdots a_{m+1}}\right)\right]_{P}
$$

about the point in $\kappa$-space for which $\kappa_{a i n}=0$ for $n \geqq 3$. The first term is given by

$$
\left(\alpha_{\nu}^{a_{1} a_{2} \cdots a_{m+1}}\right)_{P}=\left\{\phi_{a_{1}, a_{2}, \cdots, a_{m+1}}^{(\nu)}(0)\right\}_{P}
$$

which is, from (4.8),

$$
\left(\alpha_{\nu}^{\left.a_{1} \cdots a_{m+1}\right)_{P}}=\left(\frac{i}{2 \pi}\right)^{m} \int_{-\infty}^{\infty} d t_{1} \int_{-\infty}^{\infty} d t_{m} \prod_{i=1}^{m}\left(\frac{\Psi_{a_{i}}\left(t_{i}\right)}{t_{i}}\right)_{P}\left[\Psi_{a_{m+1}}^{(\nu)}\left(\xi_{m}\right)_{P}\right]_{t=0} .\right.
$$

On carrying out the operations indicated on the last factor in the integrand one finds (see appendix I)

$$
\begin{gathered}
\left(\alpha_{\nu}^{a_{\nu} \cdots a_{m+1}}\right)_{P}=C_{\nu} \int_{s} d \tau\left(\prod_{i=1}^{m} t_{i}\right)^{-1}\left[\exp \left(i x^{\prime} t-t^{\prime} A t\right)\right] H_{\nu}\left(\tau_{m}\right), \\
\left(\frac{\partial \alpha_{\nu}}{\partial \kappa_{a_{i \mu}}}\right)_{P}=\left(\frac{C_{\nu} i^{\mu}}{\mu !}\right) \int_{s} d \tau t_{i}^{\mu}\left\{\exp \left(i x^{\prime} t-t^{\prime} A t\right)\right\} H_{\nu}\left(\tau_{m}\right), \quad i<m+1, \\
\left(\frac{\partial \alpha_{\nu}}{\partial \kappa_{a_{m+1}, \mu}}\right)_{P}=\left(\frac{C_{\nu} i^{\mu}}{\mu !}\right) \int_{s} d \tau\left[\prod_{j=1}^{m} t_{i}\right]^{-1}\left\{\exp \left(i x^{\prime} t-t^{\prime} A t\right)\right\} S_{\sigma \nu}
\end{gathered}
$$

and

$$
\widetilde{S}_{\sigma \nu}=\sum_{\mu=0}^{\nu}\left(\begin{array}{c}
\nu \\
\mu
\end{array}\right) \frac{\sigma !(-1)^{\nu-\mu}}{(\sigma-\mu) !}\left(\frac{\kappa_{a_{m+1} 2}}{2}\right)^{(\nu-\mu) / 2} H_{\nu-\mu}\left(\tau_{s}\right)
$$


where

$$
C_{\nu}=\left(\frac{i}{2 \pi}\right)^{m}(-1)^{\nu}\left(\frac{\kappa_{a_{m+1}, 2}}{2}\right)^{\nu / 2} \exp \sum_{i=1}^{m+1} \kappa_{i 0} .
$$

The exponential is the matrix representation of the quadric,

$$
\begin{gathered}
i x^{\prime} t-t^{\prime} A t=-\frac{1}{2} \sum_{i=1}^{m}\left(\kappa_{a_{i}, 2}+\kappa_{a_{m+1}, 2}\right) t_{i}^{2}-\frac{1}{2} \kappa_{a_{m+1}, 2} \sum_{p>a=1}^{m} t_{p} t_{a} \\
+i\left[\sum_{i=1}^{m} \kappa_{a_{i} 1} t_{i}-\kappa_{a_{m+1} 1} \sum_{j=1}^{m} t_{i}\right], \\
\tau_{m}=\left(\frac{\kappa_{a_{m+1} 2}}{2}\right)^{1 / 2}\left(\sum_{p=1}^{m} t_{p}+\frac{i \kappa_{a_{m+1}, 1}}{\kappa_{a_{m+1}, 2}}\right)
\end{gathered}
$$

and the integral is over $m$-dimensional space.

APPENDIX I-Evaluation of $\left(\alpha_{\nu}\right)_{P}$ and $\left(\partial \alpha_{\nu} / \partial \kappa_{\sigma}\right)_{P}$

These integrals $(3.9,3.10,3.11)$ all reduce to integrals of the form

$$
\int_{\tau} d \tau \exp \left[-A f\left(x_{1}, x_{2}, \cdots, x_{n}\right)\right] x_{1}^{m_{1}} x_{2}^{m_{2}} \cdots x_{n}^{m_{n}},
$$

with $\sum m_{K}$ even if $n$ is odd and vice versa ( $m_{i}$ integers),

$$
f\left(x_{1}, x_{2}, \cdots, x_{n}\right)=\sum_{i=1}^{n} x_{i}^{2}+\frac{1}{2} \sum_{i \neq i} x_{i} x_{i} .
$$

Obviously, several integrations by parts would reduce this to an evaluation of a wellknown integral. A simpler technique is available. For example, with $s=2$ we insert the parameters $a, b$ to form:

$$
f(a, b)=\int_{-\infty}^{\infty} d x_{1} \int_{-\infty}^{\infty} d x_{2}\left(x_{1} x_{2}\right)^{-1} \exp \left[-\kappa_{2}\left(a x_{1}^{2}+x_{2}^{2}+b x_{1} x_{2}\right)\right] .
$$

One then evaluates $\int_{0}^{b} d b \partial f / \partial b$ to find

$$
f(a, b)=-2 \pi \sin ^{-1}\left(b / 2 a^{1 / 2}\right) .
$$

Setting $a=b=1$ in the derivatives of this function then gives the values of the required integrals.

Moments up to the fifth have been tabulated for $s=1$ and $s=2$. For $s=3$ only first terms for moments up to the fourth have been evaluated.

\section{References}

[1] Harald Cramér, Methods of mathematical statistics: Princeton University Press, New Jersey, 185-186 (1946).

[2] G. Doetsch, Theorie und Anwendung der Laplace Transformation, Dover Publications, New York, 101-102 (1943). 